\title{
La méthode des éléments finis stochastiques en géotechnique
}

\section{G. AUVINET}

Instituto de Ingeniería UNAM

Cd. Universitaria Apdo Postal 70-472

Coyoacán 04510 Mexico

R. MELLAH F. MASROURI

Laboratoire Environnement Géomécanique et Ouvrages École Nationale Supérieure de Géologie, INPL, Nancy

\section{J. F. RODRIGUEZ}

Instituto de Ingeniería UNAM, Mexico
Cet article présente différentes techniques permettant d'appliquer la méthode des éléments finis stochastiques (MEFS) aux ouvrages géotechniques. La MFES a pour but d'évaluer la marge d'erreur sur les résultats des modélisations numériques du comportement de ces ouvrages, compte tenu des diverses incertitudes qui affectent ces modélisations et, en particulier, de celles qui portent sur les paramètres du sol. On montre comment la méthode des éléments finis peut être couplée à des techniques probabilistes telles que la méthode de MonteCarlo ou les diverses techniques de perturbation, y compris les méthodes du « premier ordre-seconds moments » et la méthode des estimations ponctuelles. Les avantages et les inconvénients de ces diverses techniques sont examinés. L'application de la MEFS est brièvement illustrée par des analyses d'incertitude sur le champ de contraintes dans une structure simple et sur le champ des déplacements dans un remblai. Les résultats de ces analyses et d'autres similaires qui seront présentées dans de prochains articles montrent que la MEFS, malgré ses limites actuelles, permet aux ingénieurs géotechniciens d'introduire un degré de réalisme supplémentaire bien nécessaire dans les modélisations aux éléments finis.

Mots-clés : géotechnique, incertitude, méthode des éléments finis stochastiques, sols, méthode de Monte Carlo, techniques de perturbations.
NDLE: Les discussions sur cet article sont acceptées jusqu'au $1^{\text {er }}$ août 2001.
This paper presents several techmiques making it possible to apply the Stochastic Finite Element Method (SFEM) to geotechnical structures. SFEM can be used for assessing the error margin on the results of numerical modeling of such structures, taking into account the main uncertainty sources, including those concerning soil parameters. As shown in the paper, the Finite Element Method can be coupled with probabilistic techniques such as the Monte-Carlo Method and different perturbation techniques Including the First OrderSecond Moment method and the Point Estimate method. The advantages and limitations of these different techniques are discussed. The application of the SFEM is briefly illustrated by analyses of uncertainty on the stress field within a simple structure and on the displacement field within an embankment. The results of these analyses and those of similar ones that will be presented in future papers show that, in spite of its current limitations, the SFEM can help geotechnical engineers to introduce a quite necessary additional degree of realism in Finite Element modeling.

Key words : geotechnical engineering, uncertainty, stochastic finite element method, soils, Monte Carlo method, perturbation techniques. 


\section{Introduction}

L'application de l'informatique et de méthodes numériques telles que les différences finies ou les éléments finis a connu un large développement dans le domaine de la modélisation des déplacements, déformations et contraintes dans les sols et les ouvrages en terre.

Cependant, les sources d'incertitude dans les analyses aux éléments finis en géotechnique sont nombreuses. Le choix des paramètres des lois de comportement à introduire dans le calcul est particulièrement délicat. Les propriétés des sols naturels ou compactés présentent généralemient des variations spatiales importantes et les techniques directes ou indirectes utilisées pour les déterminer sont souvent peu fiables. Aux incertitudes sur les propriétés, s'ajoutent celles concernant les sollicitations, les conditions aux limites et la méthode de calcul elle-mème. On s'accorde en général à reconnaître que les incertitudes les plus graves sont celles induites par une mauvaise connaissance des propriétés des sols.

Les techniques statistiques et probabilistes permettant d'évaluer l'incertitude sur le comportement des sols et des ouvrages liée aux diverses incertitudes citées ci-dessus dans les analyses aux éléments finis portent globalement le nom de " méthode des éléments finis stochastiques n (MEFS). Comme on le montre dans ce qui suit, les différentes techniques disponibles pour la mise en cuvre de la MEFS ont leur domaine d'application, leurs limitations et leurs mérites respectifs. Les exemples présentés montrent par ailleurs que les résultats typiques obtenus par la MEFS sont souvent d'interprétation délicate.

Les premiers travaux sur l'application de la MEFS à la géotechnique ont été exposés par Cambou et Auvinet (1974, 1975, 1977). Un logiciel a été développé pour l'analyse stochastique des déplacements et des contraintes dans le sol au cours des excavations réalisées pour la construction du métro de Mexico. Une méthodologie analogue a été utilisée par Baecher et al. (1981) pour l'étude des incertitudes sur les tassements des fondations. Un logiciel de MFES a été développé par Magnan (1987) (Code de calcul PROBEF). Magnan et al. (1995) ont par la suite appliqué la MEFS à I'analyse de la consolidation uni- et bidimensionnelle des sols sous une semelle isolée. Orlandi (1996) et Bouayed (1997) ont aborcé l'analyse stochastique des barrages en terre en utilisant la méthode FOSM en élasticité linéaire et non-linéaire mais en se limitant dans ce dernier cas aux incertitudes sur les déplacements. Mellah (1999) a présenté une évaluation de l'applicabilité de la méthode au cas des analyses par éléments finis en élasto-plasticité.

Dans le domaine des écoulements en milieu poreux obéissant à la loi de Darcy, Griffith et al. (1993) ont étudié l'influence de la variabilité spatiale de la perméabilité sur l'écoulement de l'eau sous un barrage par simulation de champs stochastiques. Par ailleurs, Bencheikh (1994) et López Acosta (1999) ont appliqué la méthode des éléments finis stochastiques à l'étude des écoulements souterrains dans des milieux à perméabilité incertaine décrite au moyen de variables aléatoires.

\section{Les incertitudes dans les analyses aux éléments finis en géotechnique}

\author{
2.1
}

\section{Les principales sources d'incertitude}

Si, pour les structures, la diversité des matériaux est relativernent réduite et leurs propriétés sont maintenant assez bien connues, à l'opposé, la mécanique des sols doit s'accommoder de matériaux que la nature a dotés de caractéristiques complexes et variables dans l'espace et dans le temps et dont la mesure est délicate. De ce fait, les paramètres mécaniques que l'on doit introduire dans les calculs en géotechnique, et en particulier dans ceux réalisés par la méthode des éléments finis, sont souvent mal connus. On doit ajouter à cela les incertitudes sur les sollicitations et les conditions aux limites ainsi que l'erreur que peuvent introduire les hypothèses et approximations du modèle mécanique utilisé. Les incertitudes associées aux erreurs humaines peuvent également être extrêmement importantes mais elles échappent en général aux modélisations probabilistes ou autres.

\section{antis}

\section{Incertitudes sur les paramètres mécaniques des sols}

La connaissance des lois de comportement des sols naturels ou compactés joue un rôle fondamental dans les calculs de la géotechnique et en particulier dans les analyses aux éléments finis effectuées en vue de la détermination des champs de déplacements, de contraintes et de déformations au sein des ouvrages et de leur environnement. Les paramètres de la loi de comportement (élastique linéaire ou non-linéaire, élastoplastique, viscoélastique) sont souvent estimés à partir de l'expérience, de corrélations avec les propriétés physiques et, dans le meilleur des cas, à partir d'un nombre limité d'essais en place ou au laboratoire. Il est clair que l'on doit distinguer deux types d'incertitudes: celles qui sont associées aux variations spatiales et celles qui sont dues à la mesure ou à l'estimation des paramètres d'un élément de sol donné.

\section{- Variabilité spatiale}

Par nature, les sols sont des matériaux hétérogènes, leurs propriétés mécaniques et physiques présentent une variation souvent considérable d'un point à l'autre du milieu géotechnique étudié. La connaissance de la géologie du site ou du procédé de construction de l'ouvrage permet en général de définir des sousdomaines à caractéristiques à peu près homogènes. Il peut ne s'agir toutefois que d'une homogénéité statistique dissimulant des variations spatiales souvent très significatives. Il est donc nécessaire de reconnaître que les propriétés des sols sont des fonctions aléatoires spatiales.

\section{- Incertitudes sur les mesures et estimations des paramètres}

Les lois de comportement courantes sont des modèles qui donnent du comportement réel du sol une 
description plus ou moins satisfaisante. Dans la plupart des cas, les courbes expérimentales décrivant le comportement du sol au cours d'essais de laboratoire ou en place sont ajustées à celles prévues par modèle choisi, afin de déterminer les paramètres mécaniques du sol considéré. L'écart observé entre le comportement réel du sol et la réponse du modèle choisi est dû principalement:

- aux erreurs aléatoires commises lors des essais réalisés;

- aux erreurs systématiques dues à un biais dans la mesure, souvent inhérent à l'essai réalisé (on observe par exemple des différences systématiques entre la résistance au cisaillement mesurée au scissomètre et au triaxial) ou lié au remaniement des échantillons et autres facteurs similaires.

Un biais peut également être introduit lorsque la loi de comportement ajustée est choisie de façon trop arbitraire et surtout quand les paramètres du sol sont estimés de façon subjective (jugement d'expert) ou à partir de corrélations statistiques entre propriétés physiques et mécaniques. Ce biais, le plus souvent mal connu, donc aléatoire, peut être additif (résultat de l'essai ou de l'estimation valide à une constante aléatoire près) ou, le plus souvent, multiplicatif (erreur systématique aléatoire proportionnelle au résultat de la mesure ou de l'estimation).

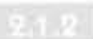

\section{Incertitudes sur les sollicitations et conditions aux limites}

Les forces extérieures et les forces de volume à prendre en compte dans les analyses géotechniques réalisées par la méthode des éléments finis, sont souvent elles aussi mal connues. Il en est ainsi des forces tectoniques ou des contraintes transmises au sol par les fondations, fréquemment estimées à partir de descentes de charges approximatives. Les conditions aux limites elles-mêmes ne peuvent souvent être représentées que de façon approchée. Les incertitudes ainsi introduites sont souvent difficiles à évaluer. Si l'on dispose des éléments nécessaires, ce type d'incertitude peut être représenté par un vecteur de variables aléatoires susceptibles d'être prises en compte dans les analyses stochastiques (Cornell, 1971).

\section{X74.}

\section{Incertitudes sur la méthode de calcul}

Il existe des méthodes théoriques pour déterminer l'erreur implicite dans les analyses aux éléments finis a priori et a posteriori (Mestat et Prat, 1999). On trouve par ailleurs dans la littérature, mais relativement dispersées, diverses études de sensibilité sur les différentes caractéristiques d'une modélisation numérique (maillages, méthode d'intégration, critère de plasticité....). En général, ces études ne sont pas réalisées de manière systématique et ne se prêtent donc guère au traitement probabiliste et statistique, qui permettrait une étude des facteurs susceptibles d'introduire un biais dans les calculs aux éléments finis. L'approche de ce problème est souvent empirique et passe par la validation des résultats sur essais, ouvrages types, et ouvrages réels. Si les éléments nécessaires sont réunis, il est toutefois possible de représenter l'erreur sur la méthode de calcul au moyen d'un terme aléatoire d'erreur multiplicatif ou additif (Cornell, 1971).

\section{1 .4}

\section{Conclusion}

Nous avons souligné l'importance que peuvent revêtir les incertitudes sur les propriétés du sol, mais aussi sur les sollicitations, les conditions aux limites et sur la validité du modèle lui-même dans les analyses aux éléments finis. Il semble qu'en géotechnique, le plus souvent, l'incertitude sur les paramètres mécaniques des matériaux considérés soit la plus importante. Dans la suite, nous nous intéresserons donc plus particulièrement à la modélisation de cette incertitude en vue de sa prise en compte dans les analyses aux éléments finis.

\section{2}

\section{Modélisation de l'incertitude sur les propriétés des sols}

\section{glot \\ Champ stochastique}

Soit $V(X)$, la valeur prise par une propriété du sol donnée au point $X$ d'un domaine $R^{F}(p=1,2,3)$. En chaque point, cette valeur, en général non ou mal connue, peut être considérée comme une variable aléatoire. L'ensemble des variables aléatoires du domaine constitue un champ stochastique qui peut être décrit au moyen des éléments suivants :

- Espérance

mathématique: $\quad \mu_{V(x)}=E[V(X)]$

- Variance : $\quad \sigma_{v x}^{2}=\operatorname{Var}[V(X)]$

- Auto-corrélation:

$R_{V}\left(X_{1}, X_{2}\right)=E\left[V\left(X_{1}\right) V\left(X_{2}\right)\right]$

- Auto-covariance :

$$
C_{w}\left(X_{1}, X_{2}\right)=E\left[\left(v\left(X_{1}\right)-\mu_{v\left(x_{1}\right)}\right)\left(v\left(X_{2}\right)-\mu_{v\left(x_{2}\right)}\right)\right]
$$

Les deux fonctions précédentes représentent le degré de dépendance linéaire entre les valeurs des propriétés mesurées en deux points différents dans le domaine considẻré.

- Coefficient d'auto-corrélation :

$$
P_{v V}\left(X_{1}, X_{2}\right)=\frac{C_{V V}\left(X_{1}, X_{2}\right)}{\sigma_{V\left(X_{1}\right)} \sigma_{V\left(X_{2}\right)}}
$$

Ce coefficient représente l'auto-covariance normalisée, adimensionnelle, dont les valeurs restent comprises entre -1 et +1 .

- Fonction de distribution cumulée de probabilité : $F_{V\left(X_{1}\right), V\left(X_{2}\right), \ldots, V_{\left(X_{n}\right)}}\left(v_{1}, v_{2}, \ldots, v_{n} ; X_{1}, X_{2}, \ldots, X_{n}\right)=P\left[\left(V\left(X_{1}\right)\right.\right.$ $\left.\left.\leq v_{1}, V\left(X_{2}\right) \leq v_{2}, \ldots, V\left(X_{n}\right) \leq v_{n}\right)\right]$

On suppose le plus souvent en pratique que le champ présente une certaine régularité. On admet qu'il existe une homogénéité statistique, quitte à subdiviser le milieu en plusieurs sous-domaines. L'hypothése simplificatrice la plus courante est celle de la stationnarité au sens large. L'espérance est considérée comme constante dans le domaine étudié, éventuellement après élimination d'une dérive, et l'on admet que l'auto- 
covariance dépend uniquement de la distance vectorielle $t$ entre les points $X_{1}$ et $X_{2}$ on a alors :

$C_{W V}\left(X_{1}, X_{2}\right)=C_{V V}\left(X_{2}-X_{1}\right)=C_{V v}\left(X_{2}-X_{1}\right)=C_{W v}(\tau)$ (ou plus simplement $C(\tau)$ )

A l'origine, cette fonction n'est autre que la variance de $V(X)$.

$$
\operatorname{Var}[V(X)]=C(O)
$$

et le coefficient d'auto-corrélation s'écrit comme suit:

$$
\rho_{w}\left(X_{1}, X_{2}\right)=\rho_{W}(\tau) \text { ou plus simplement } \rho(\tau)
$$

La représentation graphique de cette fonction reçoit souvent le nom de "corrélogramme $n$.

Sous le même formalisme mathématique, l'interprétation donnée à ce champ peut être extrêmement variable. Suivant le sens attribué au concept de probabilité, qưi doit bien sûr être clairement défini avant toute analyse probabiliste, on est amené en fait à considérer plusieurs types de champs aléatoires.

\section{a) Champs estimatifs}

Lorsque les propriétés des sols sont estimées à partir d'opinions basées principalement sur l'expérience et les observations d'un expert, le champ stochastique est de type estimatif. Ses paramètres doivent alors refléter les connaissances mais aussi l'ignorance de l'expert. Cette situation est courante en géotechnique car il n'est pas toujours possible de réaliser un nombre de mesures suffisant pour pouvoir s'affranchir d'opinions largement subjectives. Dans ce cas, les probabilités reflètent avant tout le « degré de confiance » (degree of belief) que méritent les estimations avancées par l'expert.

Au moment du projet des ouvrages en terre, on ne dispose bien entendu d'aucunes données sur la variabilité spatiale des propriétés, à l'exception du zonage général prévu par le projet lui même. Dans cette situation, qui est une de celles où la modélisation stochastique présente le plus grand intérêt, seul l'emploi d'un champ estimatif reflétant les incertitudes a priori sur les propriétés des matériaux des différentes zones est alors possible.

\section{b) Champs descriptifs de la variabilité spatiale}

Lorsque l'on dispose d'un nombre suffisant de mesures fiables sur la variabilité spatiale des propriétés du sol, le champ aléatoire peut être de type descriptif et être utilisé pour décrire les incertitudes résiduelles. Les paramètres du champ peuvent alors ètre estimés par analyse statistique des données. C'est le domaine de la géostatistique au sens qui lui a été donné durant ces dernières décennies. Dans ce cas, les probabilités doivent être interprétées en termes de fréquence relative. On soulignera que les champs stochastiques décrivant la variabilité spatiale des propriétés des sols ne sont connus que par l'intermédiaire de mesures ou d'estimations qui sont ellesmêmes entachées d'incertitude. On ne peut donc avoir accès qu'à un champ apparent dont les caractéristiques diffèrent de celles du champ réel.

Dans la pratique de la géotechnique, l'utilisation de ces champs descriptifs se heurte souvent à de sérieuses difficultés pour les raisons suivantes:

- l'estimation des paramètres du champ doit suivre les règles applicables à la prise d'échantillons représenta- tifs aléatoires ou systématiques (Cochran, 1977). Il est courant en géotechnique que le nombre de données disponibles ne soit pas suffisant pour que l'estimation soit précise ; une erreur de type statistique est alors introduite dans l'estimation:

- à l'inverse, quand les données sont nombreuses et fiables, il devient possible d'estimer les paramètres du champ avec précision mais c'est alors toute l'approche probabiliste de la variabilité spatiale qui perd de son intérêt du point de vue de l'ingénieur ;

- la corrélation entre les valeurs du champ associées à deux points du milieu n'est pas une propriété intrinsèque de ces deux points. Elle dépend bien entendu du domaine (population) dans lequel elle est définie et duquel elle ne peut être dissociée. Ainsi, dans un milieu fortement contrasté du point de vue stratigraphique, les propriétés de deux points appartenant à une même couche peuvent être fortement corrélées, mais ne plus l'être du tout dans une analyse de corrélation ne portant plus que sur la couche ou ils se trouvent. Un sondage trop court ou des mesures faites dans un seul plan horizontal ou tout autre zone particulière du sol peuvent donner une image totalement incorrecte de la variabilité et de la structure de corrélation de l'ensemble du milieu :

- à défaut de mesures, il est parfois possible d'estimer l'ordre de grandeur des distances d'auto-corrélation. On dispose maintenant de nombreux résultats obtenus sur différents types de sols mis en place par diverses techniques et concernant en particulier la portée ou distance d'influence de la corrélation (Rossa, 1988; Favre, 1987 ; Cherubini, 1993). Il est toutefois indispensable que les conditions géotechniques du site étudié soient vraiment similaires à celles des sites où les mesures ont été réalisées pour que ces valeurs puissent être adoptées ;

- remarquons par ailleurs que les champs stochastiques tridimensionnels ne peuvent être pris en compte dans les analyses courantes aux éléments finis en déformation plane. En effet ce type d'analyse suppose une homogénéité parfaite dans la direction perpendiculaire au plan de l'analyse, ce qui contredit l'hypothèse de variation aléatoire spatiale.

\section{c) Champs mixtes}

En pratique, la situation est souvent intermédiaire entre les deux précédentes. Les propriétés des sols sont définies par une combinaison d'opinions (probabilités a priori) et de résultats expérimentaux qui peuvent être introduits dans une analyse bayésienne explicite ou implicite permettant de définir des probabilités a posteriori (tenant compte des mesures). L'interprétation donnée au concept de probabilité devient dans ce cas plus ambigu mais ce concept reste malgré tout utile dans la pratique (Divtlevsen, 1996).

\section{a 203}

\section{Discrétisation pour les analyses aux éléments finis}

Pour introduire l'incertitude sur les propriétés des sols dans un modèle de calcul, il est nécessaire de passer de la représentation du champ aléatoire continu à un nombre limité de variables aléatoires. C'est ainsi que, pour les calculs aux éléments finis, on devra finalement associer à chaque élément ou groupe d'éléments correspondant à un volume de sol donné, des 
paramètres mécaniques aléatoires représentatifs. Le problème de la définition de ces valeurs représentatives est délicat car il est souvent de nature tout autant mécanique que statistique. Les méthodes de discrétisation les plus courantes ignorent en fait l'aspect mécanique du problème qui ressort des techniques d'homogénéisation.

Soit $V(X) ; X \in \Omega$, un champ aléatoire multidimensionnel, défini dans un domaine $\Omega$ et représenté par son espérance $\mu_{\mathrm{V}}(X)$, sa variance $\sigma_{\mathrm{V}}^{2}(X)$ et son coefficient d'autocorrelation $\rho_{v v}\left(X, X^{\prime}\right)$. V est le vecteur des variables qui permettent de représenter le champ stochastique, défini par un vecteur moyen $\mu$ et une matrice de covariance $T_{1}$. Il existe diverses méthodes de discrétisation reliant $V(X)$ à $V(D e r$ Kiureghian et al., 1988). Nous n'examinerons ici que les plus simples.

\section{- Méthode des points moyens}

La valeur du champ dans le domaine $\Omega_{e}$ d'un élément ou groupe d'éléments est décrite par une simple variable aléatoire représentant la valeur au point central du sous-domaine considéré :

$$
\tilde{V}(X)=V\left(X_{c}\right) \operatorname{avec} X \in \Omega_{e}
$$

Le vecteur $V$ est formé par toutes les variables aléatoires $V(X)$ des sous-domaines considérés. La réalisation du champ ainsi définie est une fonction en escalier présentant des discontinuités aux frontières des éléments. La moyenne et la matrice de covariance du vecteur sont données en fonction de la moyenne, de la variance et des coefficients d'auto-corrélation du champ, évalués au centre de gravité des sousdomaines.

\section{- Méthode des moyennes spatiales}

Cette méthode a été proposée par Cornell (1971), puis reprise par Vanmarcke et al. (1983). Le champ est décrit à l'intérieur du sous-domaine considéré par la moyenne spatiale du champ :

$$
\bar{V}(X)=\bar{V}_{e}=\frac{\int_{\Omega_{e}} V(X) d \Omega_{e}}{\int_{\Omega_{e}} d \Omega_{e}} \operatorname{avec} X \in \Omega_{e}
$$

L'ensemble des valeurs moyennes $\bar{V}_{e}$ des variables aléatoires forme le vecteur $V$; les valeurs statistiques $\mu$ et $T_{j y}$ (espérance et matrice de covariance) du vecteur $V$ sont obtenues par intégration des moments du champ aléatoire.

La variance de la valeur moyenne d'une propriété aléatoire dans un domaine donné tend à diminuer quand les dimensions du domaine considéré augmentent (sauf dans le cas trivial de la corrélation parfaite). Dans la littérature, ce phénomène est connu sous le nom de $\alpha$ réduction de variance $v$. Dans la mesure où l'on a recours à des moyennes spatiales pour les calculs aux éléments finis, les incertitudes résiduelles les plus significatives sont donc souvent celles correspondant aux biais des tests ou des corrélations, qui eux ne souffrent pas de réduction de variance, ce qui peut justifier dans de nombreux cas, au moins en géotechnique, l'abandon de la modélisation par champ stochastique et son remplacement par une modélisation plus simple par variables aléatoires.

- Valeurs ponctuelles et moyennes spatiales définies dans des champs conditionnels

Dans les paragraphes précédents, on a supposé que le modèle stochastique de l'incertitude spatiale avait pu être établi à partir de certaines données ponctuelles ou continues (sondages) et on a examiné ses possibilités d'utilisation pour les modélisations stochastiques dans les analyses aux éléments finis. Cette approche ignore le fait que l'existence de ces données réduit considérablement l'incertitude au voisinage des points où elles ont été obtenues. Il est évident que l'on peut faire mieux et estimer les paramètres à attribuer aux éléments finis ou groupes d'éléments finis en prenant en compte directement les résultats des mesures. C'est l'objectif des techniques d'estimation conditionnelle, telles que le krigeage (Matheron, 1971) qui peuvent être utilisées pour associer à chaque point du milieu une espérance et une variance conditionnelle de la propriété considérée ou pour attribuer à chaque sousdomaine considèré (élément fini ou groupe d'éléments finis) une espérance et variance conditionnelle de la valeur moyenne de cette propriété sur ce sousdomaine.

\section{Conches \\ Conclusion}

Les mesures et estimations et la variabilité spatiale des propriétés des sols introduisent une incertitude importante dans les analyses aux éléments finis. L'incertitude sur les propriétés des sols peut être représentée par des champs stochastiques. Néanmoins, dans la pratique, la description de l'incertitude devra finalement ètre réalisée au moyen d'un nombre limité de variables aléatoires.

\section{3}

\section{La méthode des éléments finis stochastiques}

Le couplage entre les méthodes probabilistes et la méthode des éléments finis a donné naissance à la (cméthode des éléments finis stochastiques » (MEFS). Les techniques permettant d'appliquer la méthode des éléments finis stochastiques (MEFS), peuvent généralement être divisées en deux catégories (Teigen et al. 1991) : les méthodes des perturbations et les méthodes de simulation. Par ailleurs, la méthode peut être couplée avec un calcul de fiabilité (Flores, 1994; Lemaire, 1997). La littérature consacrée au développement de ces méthodes et, dans une moindre mesure, à leurs applications dans différents domaines est abondante. Nous citerons entre autres : Vanmarcke et al. (1983) et Bittnar (1996).

\section{1}

\section{Méthode des perturbations}

La méthode des perturbations est basée sur une représentation simplifiée des fonctions de distribution 
de probabilité de fonctions de variables aléatoires, au moyen des nièmes premiers moments (le nombre dépendant de la technique utilisée). L'estimation de ces premiers moments est obtenue en ajoutant à un terme central moyen les effets " perturbateurs » introduits par chaque variable aléatoire considérée séparément.

\section{$3 i t-1$}

\section{Méthode classique des perturbations (Mathews, 1964)}

La méthode est basée sur l'approximation de la densité de probabilité d'une fonction de variable aléatoire à partir du développement limité en série de Taylor de cette fonction.

La résolution du système d'équations de base de la méthode des éléments finis en élasticité linéaire permet de déterminer le vecteur du champ des déplacements et par conséquent ceux des contraintes et des déformations

$$
\langle U\rangle=[K]^{-1}\{F \mid
$$

avec:

$|U\rangle$ : vecteur du champ de déplacements aux nceuds du système :

$[F]$ : vecteur des forces extérieures et volumiques appliquées aux nceuds ;

$[K]$ : matrice de rigidité fonction du vecteur de variables aléatoires $\mathrm{x}=\left(x_{i}, x_{2}, \ldots, x_{n}\right)^{\text {r }}$ qui représentent les aléas du système.

Si les aléas sont assez petits et présentent une moyenne nulle, la matrice de rigidité peut se mettre sous la forme :

$$
[K]=\left[K^{0}\right]+\sum_{i=1}^{n}\left[K_{i}^{l}\right] x_{i}+\frac{1}{2} \sum_{j=1}^{n} \sum_{j=1}^{n}\left[K_{i j}^{\prime \prime}\right] x_{i} x_{j}+\ldots
$$

$n$ : nombre de variables aléatoires,

$\left[K^{\circ}\right]$ : matrice de rigidité évaluée en $\mathrm{x}=\left(x_{1}, x_{2}, \ldots, x_{n}\right)^{\mathrm{r}}=0$. $\left[K^{\prime}\right]$ et $\left[K_{b}^{\prime \prime}\right]$ : dérivées partielles de la matrice de rigidité $[K]$, définies par:

$$
\left[K_{i}^{\prime}\right]=\left.\frac{\partial[K]}{\partial x_{i}}\right|_{x \in 0} ;\left[K_{i}^{\prime \prime}\right]=\left.\frac{\partial^{2}[K]}{\partial x_{i} \partial x j}\right|_{x=0}
$$

Le vecteur du champ de déplacements $[U]$ prend alors la forme suivante:

$$
\{U\}=\left\{U^{0}\right\}+\sum_{i=1}^{n}\left\{U^{i}\right\} X_{i}+\frac{1}{2} \sum_{i=1}^{n} \sum_{i=1}^{n}\left[U_{i j}^{I l}\right] x_{i} \cdot x_{j}+\ldots
$$

Les vecteurs des coefficients $\left\{U^{\top},\left\{U^{\prime}\right\},\left\{U_{i j}^{\prime \prime}\right\}\right.$ sont évalués après avoir résolu le système d'équations suivant :

$$
\begin{gathered}
\left|U^{0}\right|=\left[K^{0}\right]^{-1}\left|F^{0}\right| \\
{\left[U_{i}^{\prime}\right]=\left[K^{0}\right]^{-1}\left(\left\{F^{\prime}\right\}-\left[K_{i}^{\prime}\right]\left\{U^{0}\right\}\right)}
\end{gathered}
$$

$\left[U_{i j}^{\prime \prime}\right]=\left[K^{0}\right]^{-1}\left(\left\{F_{i j}^{\prime \prime}\right\}-\left[K_{i}^{i}\right]\left\{U_{j}^{\prime}\right\}-\left[K_{j}^{i}\right]\left\{U_{i}^{\prime}\right\}-\left[K_{i j}^{\prime \prime}\right]\left\{U^{0}\right\}\right)$
Une fois $\left[K^{0}\right]^{-1}$ déterminée, on peut évaluer tous les déplacements $\left\{U^{\%}\right),\left\{U_{i}^{l}\right\},\left\{U_{i j}^{\| l}\right\}$. De la même façon, on peut déterminer les vecteurs du champ des contraintes $\{\sigma \mid$ et du champ des déformations $\{\varepsilon\}$ à partir des équations de la théorie d'élasticité.

La détermination des deux premiers moments de la réponse et de leurs intervalles de confiance dépend du degré d'approximation de la fonction considérée :

Si l'approximation souhaitée est du premier ordre, le vecteur du champ des déplacements s'écrit comme suit :

$$
\{U\}=\left\{U^{0}\right\}+\sum_{i=1}^{n}\left\{U_{i}^{1}\right\} x_{i}
$$

avec : $E^{1}[U]=\left\{U^{0}\right\}$

$$
\begin{aligned}
& \text { et } \left.\operatorname{Cov}^{t}[U, U]=E\left[\left(\{U\}-E^{\prime}[U]\right)(\{U\})-E^{\prime}[U]\right)\right] \\
& \left.=\sum_{i=1}^{n} \sum_{i=1}^{n}\left\{U_{i}^{\prime}\right\}\left\{U^{\prime}\right\}\right\}\left[x_{i}, x_{j}\right]
\end{aligned}
$$

Si l'approximation est du deuxième ordre, le développement en série de Taylor jusqu'au troisième terme nous permet d'écrire :

$$
\{U\}=\left\{U^{0}\right\}+\sum_{i=1}^{n}\left\{U_{i}^{i}\right\} x_{i}+\frac{1}{2} \sum_{i=1}^{n} \sum_{j=1}^{n}\left[U_{i j}^{u}\right] x_{i}, x_{j}+\ldots
$$

avec : $E^{I I}[U]=E^{I}[U]+\frac{1}{2} \sum_{i=1}^{n} \sum_{j=1}^{n}\left[U_{i j}^{l l}\right] x_{i} \cdot x,+\ldots$

$$
\begin{aligned}
& \operatorname{Cov}^{\prime \prime}[U, U]=\operatorname{Cov}^{\prime}[U, U]+\frac{1}{4} \sum_{i=1}^{n} \sum_{j=1}^{n} \sum_{k=1}^{n} \sum_{i=1}^{n}\left\{U_{i}^{H}\right\}\left\{U_{k i}^{H}\right] \\
& \left(E\left[x_{i}, x_{l}\right] E\left[x_{j}, x_{k}\right]+E\left[x_{i}, x_{k}\right] E\left[x_{j}, x_{l}\right]\right)
\end{aligned}
$$

L'application de cette méthode demande bien entendu le calcul de toutes les dérivées partielles premières et secondes de la matrice de rigidité.

\section{1.?}

\section{Méthode du premier ordre - seconds moments (FOSM; Cornell, 1971)}

La méthode FOSM, proposée par Cornell (1971), est également basée sur le développement en série de Taylor. Le caractère aléatoire est analysé en termes d'espérance mathématique, de variance et de covariances des paramètres.

Le principe de cette méthode est d'approximer la densité de probabilité d'une fonction d'une variable aléatoire, $g\left(x_{i}\right)$, en remplaçant cette dernière par les deux ou trois premiers termes de son développement en série de Taylor au voisinage de la moyenne de $x_{i}$. 


$$
\begin{aligned}
& g\left(x_{i}\right)=g\left(\mu_{x_{i}}\right)+\left(x_{i}-\mu_{x_{i}}\right)\left[\frac{\partial g\left(x_{i}\right)}{\partial x_{i}}\right]_{\mu_{x_{i}}} \\
& +\frac{\left(x_{i}-\mu_{x_{i}}\right)^{2}}{2}\left[\frac{\partial^{2} g\left(x_{i}\right)}{\partial x_{i}^{2}}\right]_{\mu_{x_{i}}}+\ldots
\end{aligned}
$$

$\mu_{x} i$ : la valeur moyenne de la variable aléatoire $x_{i}$ On peut distinguer les cas suivants :

Dans le cas d'une fonction $y=g(x)$ d'une seule variable aléatoire $x$ (et éventuellement d'autres variables non-aléatoires), l'expression (23) prend la forme suivante:

$$
\begin{aligned}
& y=g(x)=g\left(\mu_{x}\right)+\left(x-\mu_{x}\right)\left[\frac{\partial g(x)}{\partial x}\right]_{\mu_{x}} \\
& +\frac{\left(x-\mu_{x}\right)^{2}}{2}\left[\frac{\partial^{2} g(x)}{\partial x^{2}}\right]_{\mu_{x}}+\ldots
\end{aligned}
$$

et :

$$
\begin{gathered}
E[y]=E[g(x)] \approx g\left(\mu_{x}\right)+\frac{\sigma_{x}^{2}}{2}\left[\frac{\partial^{2} g(x)}{\partial x^{2}}\right]_{\mu_{x}} \\
\operatorname{Var}[y]=\operatorname{Var}[g(x)]=\sigma_{x}^{2}\left[\frac{\partial g(x)}{\partial x}\right]_{\mu_{x}}^{2}
\end{gathered}
$$

Si la fonction dépend de plusieurs variables aléatoires $y=g\left(x_{\gamma}, x_{z} \ldots, x_{n}\right)$, le développement en série de Taylor donne:

$$
\begin{aligned}
& E[y]=E\left[g\left(x_{1}, x_{2}, \ldots, x_{n}\right)\right] \approx g\left(\mu_{x+1}, \mu_{x_{2}}, \ldots, \mu_{x_{n}}\right) \\
& +\frac{1}{2} \sum_{j=1}^{n} \sum_{j=1}^{n}\left[\frac{\partial^{2} g\left(x_{k}\right)}{\partial x_{j} \partial x_{j}}\right]_{\mu_{x k} \mu_{x j}} \operatorname{Cov}\left[x_{i}, x_{j}\right] \\
& \operatorname{Var}[y]=\operatorname{Var}\left[g\left(x_{1}, x_{2}, \ldots x_{n}\right)\right] \approx \sum_{i=1}^{n} \sum_{j=1}^{n}\left[\frac{\partial g\left(x_{k}\right)}{\partial x_{i}}\right]_{i_{x_{k}}}
\end{aligned}
$$$$
\left[\frac{\partial g\left(x_{k}\right)}{\partial x_{j}}\right]_{\mu_{x_{k}}} \operatorname{Cov}\left[x_{p}, x_{j}\right]
$$

avec $n$ : nombre total de variables aléatoires.

La méthode se généralise pour les fonctions multiples de plusieurs variables aléatoires.

Pour chaque fonction :

$$
\begin{array}{r}
E\left[y_{k}\right]=E\left[g_{k}\left(x_{1}, x_{2}, \ldots, x_{n}\right)\right] \approx g_{k}\left(\mu_{x_{j}}, \mu_{x_{2}}, \ldots, \mu_{x_{n}}\right) \\
\operatorname{Var}\left[y_{k}\right] \approx \sum_{i=1}^{n} \sum_{j=1}^{n}\left[\frac{\partial g_{k}}{\partial x_{i}}\right]_{\mu_{x_{i}}}\left[\frac{\partial g_{k}}{\partial x_{j}}\right]_{\mu_{x}} \operatorname{Cov}\left[x_{i}, x_{j}\right]
\end{array}
$$

et :

$$
\operatorname{Cov}\left[y_{k}, y_{i}\right]=\sum_{i=1}^{n} \sum_{j=1}^{n}\left[\frac{\partial g_{k}}{\partial x_{i}}\right]_{\mu_{x_{i}}}\left[\frac{\partial g_{1}}{\partial x_{j}}\right]_{\mu_{x_{j}}} \operatorname{Cov}\left[x_{j}, x_{j}\right]
$$

Remarquons que cette méthode ne permet d'obtenir la forme exacte de la densité de probabilité que dans le cas où celle-ci est gaussienne.

\section{- Application de la méthode FOSM en élasticité linéaire (Cambou, 1977)}

Pour un milieu continu et isotrope et suivant une loi de comportement élastique linéaire, le principe de base des éléments finis en élasticité est la résolution du système d'équations (12):

$$
[U]=\left[K^{-1}\left(x_{1}, x_{2} \ldots, x_{n}\right]\right]|F\rangle=\left[g\left(x_{1}, x_{2}, \ldots, x_{n}\right)\right]
$$

Il est possible d'estimer les premiers moments, ainsi que les covariances des divers résultats de l'analyse, en se basant sur le développement en série de Taylor d'ordre 2 .

$$
\begin{aligned}
& E\left[U_{k}\right]=E\left[g_{k}\left(x_{1}, x_{2}, \ldots, x_{n}\right)\right] \approx g_{k}\left(\mu_{x_{1}}, \mu_{x_{2}}, \ldots, \mu_{x n}\right. \\
& \operatorname{Var}\left[U_{k}\right]=\sum_{i=1}^{n} \sum_{j=1}^{n}\left[\frac{\partial g_{k}}{\partial x_{i}}\right]_{\mu_{x_{i}}}\left[\frac{\partial g_{k}}{\partial x_{j}}\right]_{\mu_{x_{j}}} \operatorname{cov}\left[x_{i}, x_{j}\right]
\end{aligned}
$$

$$
\operatorname{Cov}\left[U_{k}, U_{j}\right]=\sum_{j=1}^{n} \sum_{j=1}^{n}\left[\frac{\partial g_{k}}{\partial x_{i}}\right]_{\mu_{x,}}\left[\frac{\partial g_{j}}{\partial x_{j}}\right]_{\mu_{x j}} \operatorname{Cov}\left[x_{1}, x_{j}\right]
$$

En dérivant les équations du système, on obtient : $\left\langle\mathrm{F}_{\mathrm{f}}\right]=[\mathrm{K}]\left(\mathrm{U}_{\mathrm{f}}\right)=\left(\mathrm{U}_{\mathrm{f}}\right]$

avec : $\left\{U_{f}\right\}=\frac{\partial\{U\}}{\partial x_{i}}$ et $\left\{F_{f}\right\}=-\frac{\partial\{K\}}{\partial x_{i}}\{U\}$

$\{U\rangle$ et $\langle F\rangle$ définissent respectivement des déplacements et forces fictives qui peuvent être utilisés pour obtenir les dérivées partielles des déplacements par rapport à chaque paramètre aléatoire en ayant recours au même algorithme que pour la résolution du système (12). Ces dérivées partielles peuvent alors être insérées dans les équations (32) à (34).

On voit que la méthode se ramène en fait à celle des perturbations classiques.

De la même façon, pour l'estimation des incertitudes sur les contraintes, on obtient, en dérivant le système d'équations général de l'élasticité par rapport aux caractéristiques aléatoires du milieu :

$$
\frac{\partial\{\sigma\}}{\partial x_{i}}=\frac{\partial[D][B]}{\partial x_{i}}\{U\}+[D][B] \frac{\partial\{U\}}{\partial x_{i}}
$$

Avec :

$|\sigma|$ : tenseur des contraintes ;

$[D]$ : matrice d'élasticité ;

$[B]$ : matrice géométrique.

Pour l'estimation des incertitudes sur les déformations, on obtient :

$$
\frac{\partial\{\varepsilon\}}{\partial x_{i}}=\frac{\partial[B]}{\partial x_{i}}\{U\}+[B] \frac{\partial\{U\}}{\partial x_{i}}
$$


Les dérivées des contraintes et des déformations obtenues à partir de ces équations peuvent être insérées dans des développements de Taylor analogues à ceux des déplacements. Ces différentes dérivées peuvent êtres déterminées explicitement en différenciant la matrice de rigidité $[K]$, la matrice $[B]$ et la matrice $[D]$ [B] par rapport aux variables aléatoires $x$ (Bouayed, 1997).

- Application de la méthode FOSM en présence de non-linéarités géométriques ou mécaniques

Si des non-linéarités géométriques ou mécaniques sont présentes dans les modèles utilisés, la détermination des déplacements en chaque nœud par la méthode des élêments finis se fait de manière itérative. Le calcul explicite des différentes dérivées des fonctions du développement de Taylor devient impossible. La méthode FOSM reste cependant applicable. II devient alors nécessaire de calculer les dérivées des dévelop. pements de Taylor numériquement. A cet effet, différents auteurs ont proposé des méthodes numériques qui permettent de calculer les différentes dérivées d'une manière approchée. Les plus utilisées sont celles des approximations d'Evans (1967) et la méthode des rapports polynomiaux (Chowdury et al. 1993). Cette dernière méthode a été utilisée dans de nombreuses études et a donné des résultats satisfaisants, tant pour des analyses de sensibilité (Orlandi, 1996; Bouayed, 1997) que pour des analyses de fiabilité (Nechnech, 1994; Benmansour, 1996). On doit souligner cependant que, dans certains cas, la méthode, comme toute technique numérique, peut se heurter localement à certaines difficultés si la fonction implicite reliant les résultats de l'analyse numérique et les paramètres aléatoires n'a pas un comportement régulier. Remarquons aussi que les méthodes numériques pour le calcul des dérivées présentent par rapport aux méthodes de dérivation explicite, l'avantage de pouvoir être appliquées de façon " externe " au code de calcul par éléments finis, sans qu'il soit nécessaire de modifier ce code.

\section{Méthode des approximations ponctuelles}

Rosenblueth (1975) a présenté une méthode qui permet d'estimer les premiers moments d'une fonction de variables aléatoires, en connaissant seulement les trois premiers moments de chaque variable et se basant sur une approximation au moyen de valeurs ponctuelles, c'est-à-dire en utilisant une distribution de probabilité discrète à mêmes moments. Il a montré qu'une formulation générale de la méthode est possible avec un nombre quelconque de points d'estimation pour chaque variable aléatoire. La démarche générale est représentée sur la figure 1.

La méthode devient malheureusement trop lourde dès que le nombre de variables aléatoires dépasse la dizaine, surtout s'il s'agit de variables corrélées entre elles.

Dans la même ligne, Bolle (1988) a proposé une méthode dite des " perturbations indépendantes " basée sur la discrétisation en trois points de la fonction de distribution de probabilité d'une variable aléatoire et faisant appel au développement limité en série de Taylor et à la transformation des variables de base en variables principales non corrélées. Les premiers moments de la distribution de la fonction $f(x)$ sont

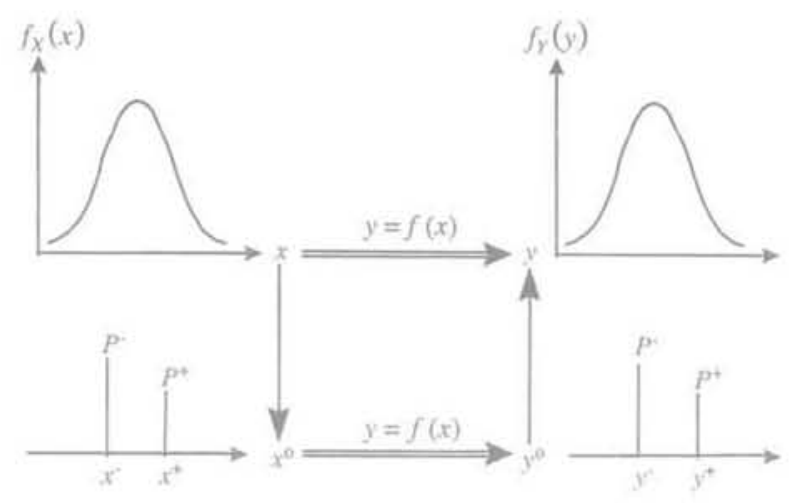

FG.1 Approximation biponctuelle de Rosenblueth Rosenblueth's two-point estimates method.

déterminés en ajoutant à la valeur de la fonction calculée au point central de la distribution discrétisée la somme des différents termes dispersants, ou perturbateurs, liés à chaque variable aléatoire indépendante (additivité des effets de chaque variable aléatoire). La méthode des perturbations indépendantes est très avantageuse si le nombre de variables considérées est important.

3.2

\section{Méthodes de simulation}

Pour se libérer des contraintes liées à l'utilisation des méthodes de transformation analytiques et numériques disponibles, dont le champ d'application est parfois trop restreint et qui sont difficiles à mettre en œuvre, il est toujours possible d'avoir recours aux techniques de simulation basées sur la méthode de MonteCarlo. Un jeu de valeurs des variables aléatoires représentant les champs stochastiques est obtenu au hasard. mais en respectant les densités de probabilité et les corrélations, et l'analyse numérique du problème est effectuée. On répète cette opération jusqu'à ce qu'une tendance statistique se définisse et permette de juger de la dispersion des résultats. Si le vecteur de variables aléatoires considéré est à composantes indépendantes, la simulation se fait pour chacune des composantes. Si le vecteur est à composantes dépendantes, on peut se ramener au cas simple précédent, en utilisant la méthode de Cholesky généralisée qui permet de diagonaliser la matrice de covariance. Cette méthode n'est valable que si le vecteur de variables aléatoires est gaussien. Dans le cas contraire, il existe dans la littérature des méthodes de simulation propres à chacune des lois utilisées. On peut par ailleurs se ramener au cas gaussien en appliquant différentes transformations (Fogli, 1980).

L'inconvénient majeur de la méthode de MonteCarlo est sa convergence extrêmement lente. Plusieurs techniques existent pour déterminer le nombre de simulations nécessaires pour une approximation fixée par un intervalle de confiance. Parmi les méthodes les plus pratiques, citons la méthode classique de convergence (intervalle de confiance d'une valeur moyenne espérée) et la méthode de Chebishev. 


\section{Applications}

Pour illustrer les techniques exposées dans les paragraphes précédents nous nous limiterons à présenter deux exemples simples.

\section{1}

\section{Analyse stochastique d'une structure simple}

Considérons une plaque de chant sur deux appuis, l'un fixe et l'autre glissant sur un plan horizontal, et soumise à une force localisée (Fig. 2). La plaque est constituée par deux matériaux à module d'Young aléatoire, mais à même espérance et avec un coefficient de variation (écart type divisé par espérance) $\mathrm{CV}=10 \%$ et corrélés négativement $(\rho=-1)$.

Les diagrammes de la figure 3 représentent l'espérance des contraintes horizontales et verticales et des cisaillements (colonne de gauche), et leur écart type (colonne de droite) obtenu para la méthode FOSM.

On est frappé par la complexité de la répartition spatiale des incertitudes. Celle-ci obéit en effet à de multiples facteurs dont les effets ne sont pas toujours mis en valeur de façon suffisamment précise par la discrétisation en éléments finis ni par l'algorithme d'interpolation (Surfer) utilisé :

- conditions aux limites concernant les contraintes (pas de contraintes verticales sur les faces horizontales de la

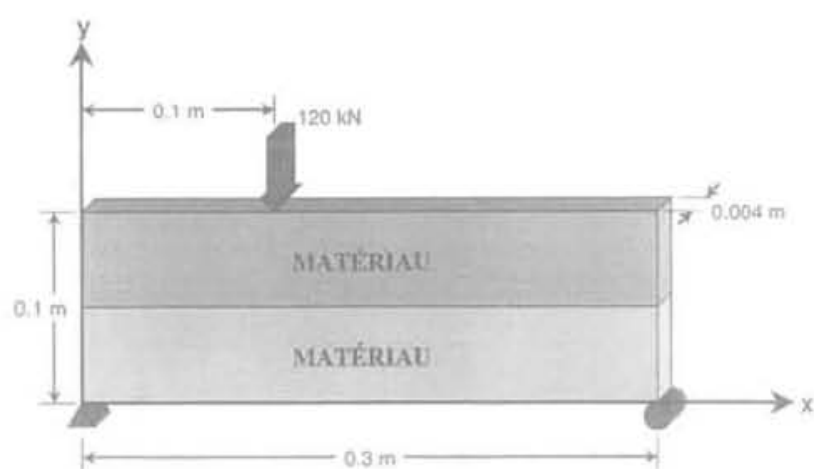

FG. 2. Plaque sur deux appuis constituée de deux matériaux différents.

Plate on two supports made of two different materials.

plaque, pas de contraintes horizontales sur les côtés verticaux, pas de cisaillements sur tous les côtés de la plaque). Ces conditions aux limites éliminent localement l'incertitude:

- perte d'homogénéité lors des variations aléatoires des deux modules, créant des sollicitations d'interaction parasites à l'interface, qui amplifient les incertitudes initiales :

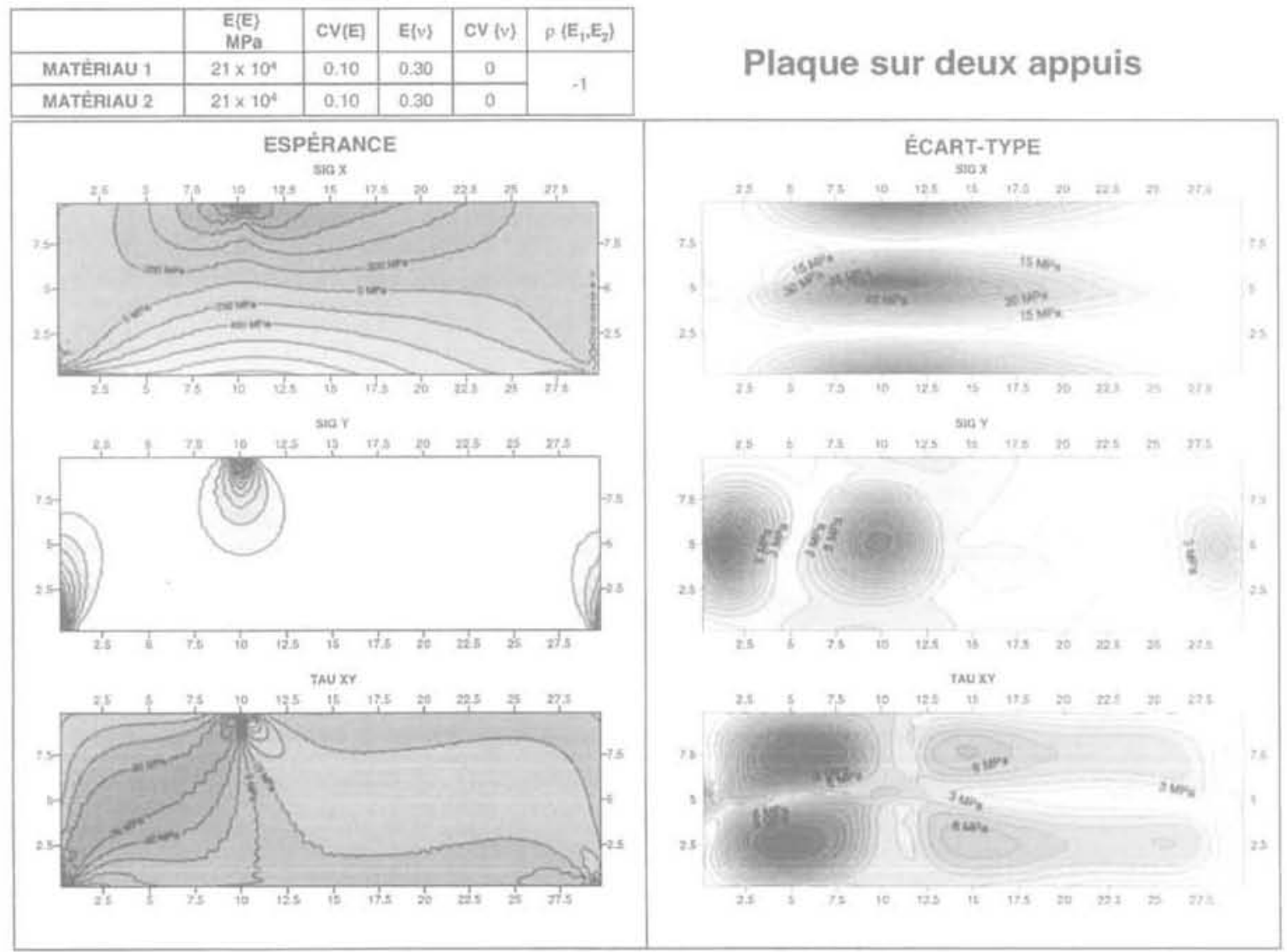

FIG. 3 Espérance et écart type des contraintes verticales (sigy), horizontales (sigx) et de cisaillement (tauxy). Expected value and standard deviation of vertical (sigy), horizontal (sigx) and shear stresses (tauxy). 
- effets de moyenne atténuant l'incertitude au voisinage de l'interface (voir cisaillement).

Seule la technique des éléments finis permet de saisir ce comportement complexe des incertitudes, difficile à prévoir a priori et d'interprétation délicate.

\section{2}

\section{Analyse stochastique d'un remblai}

Pour l'analyse des structures en terre, telles que les remblais et barrages en terre, des analyses ont pu ètre réalisées en utilisant la méthode de Monte-Carlo et la méthode FOSM. La méthode FOSM peut être mise en œuvre en procédant au calcul numérique des dérivées par la méthode des quotients polynomiaux, ce qui permet de ne pas modifier les logiciels classiques aux éléments finis tels que FEADAM84 ou CESAR-LCPC. L'emploi de ces logiciels comme sous-programmes d'une analyse d'incertitude pose toutefois de redoutables problèmes de couplage. Pour illustrer l'application de ces méthodes, nous présenterons certains résultats concernant les déplacements dans un remblai en cours de construction.

On considère ici un remblai constitué par deux matériaux mis en place par couches (Fig. 4). On admet que les deux matériaux suivent une loi non-linéaire simple de type Kondner-Duncan (Duncan, 1984). L'espérance des valeurs des neuf paramètres de cette loi est indiquée dans le tableau. Par simplicité, on a admis ici que ces paramètres présentaient tous un coefficient de variation de $10 \%$. Pour un matériau donné, les valeurs des coefficients de corrélation entre paramètres ont pu être estimées par une analyse statistique de la base de données de Duncan (1984). Pour des matériaux différents, les corrélations considérées sont subjectives et cherchent à refléter le fait que les mêmes doutes existent sur les mêmes paramètres. La matrice de corrélation finalement utilisée pour les deux matériaux est présentée sous forme de tableau (tableau I).

La modélisation par éléments finis permet de simuler la construction par couches (non-linéarité géométrique). L'incertitude sur les résultats est évaluée par Monte-Carlo et par FOSM. Le programme en éléments finis stochastiques utilisé fournit l'espérance (Fig. 5) et le coefficient de variation des déplacements verticaux dus à l'incertitude sur tous les paramètres du modèle. On observe que les résultats obtenus par FOSM (Fig. 6) et par Monte-Carlo (Fig. 7) sont analogues. Les irrégularités du champ des coefficients de variation montrent par ailleurs que pour une analyse en éléments finis stochastiques, le maillage doit généralement être plus fin que pour une analyse ordinaire.

\section{5}

\section{Conclusion}

La méthode des éléments finis stochastiques présente un intérêt particulier pour les ingénieurs géotechniciens car elle permet d'évaluer l'influence des incertitudes affectant les propriétés des matériaux sur la validité des résultats de ce type d'analyse numérique. Elle peut être employée pour obtenir les écarts types et les coefficients de variation et pour définir des intervalles de confiance sur les résultats de la modélisation tels que les déplacements, les déformations et les contraintes, Comme nous l'avons montré, malgré ses limites actuelles, la MEFS permet donc aux ingé-

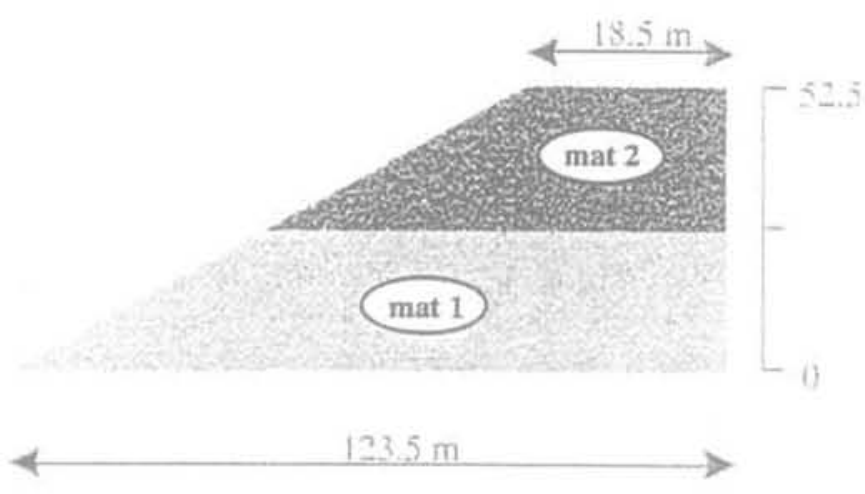

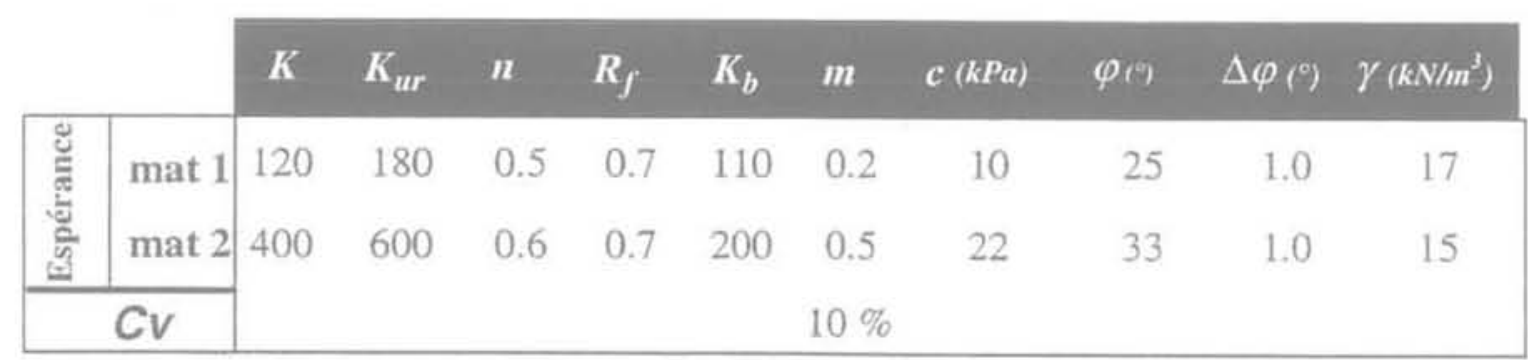

FGG 4 Remblai construit par couches et constitué de deux matériaux différents. Embankment constructed placing successive layers of two different materials. 
TABLEAUI Matrice de corrélation des propriétés des deux matériaux Correlation matrix of the two materials.

\begin{tabular}{|c|c|c|c|c|c|c|c|c|c|c|c|c|c|c|c|c|c|c|}
\hline & & & & & $\mathrm{Ma}$ & atériau 1 & & & & & & & & latérie & au 2 & & & \\
\hline & K & $K_{\mu t}$ & B & $R_{f}$ & $K_{b}$ & $m$ & Pa) & p(i) $\Delta c$ & $\left({ }^{7}\right)$ & K & $K_{u r}$ & $n$ & $R_{f}$ & $K_{\mathrm{p}}$ & $m c$ & $c(\mathrm{kPa})$ & $\varphi\left({ }^{\circ}\right)$ & $\left.\Delta \varphi f^{\circ}\right)$ \\
\hline K & 1 & 0,9 & $-0,7$ & $-0,1$ & 0,5 & $-0,2$ & $-0,2$ & 0,2 & 0,4 & 0,7 & 0,6 & $-0,4$ & 0 & 0,3 & $-0,1$ & 0 & 0 & 0 \\
\hline $\mathrm{K}_{u r}$ & 0,9 & 1 & -0.6 & $-0,1$ & 0,6 & $-0,3$ & $-0,2$ & 0,2 & 0,1 & 0,6 & 0,7 & $-0,4$ & 0 & 0,4 & 0 & 0 & 0 & 0 \\
\hline$n$ & $-0,7-$ & -0.6 & 1 & $-0,2$ & $-0,4$ & 0,3 & 0.1 & 0,2 & 0 & $-0,4$ & $-0,4$ & 0,7 & 0 & 0 & 0 & 0 & D & 0 \\
\hline $\mathrm{R}_{1}$ & $-0,1-$ & -0.1 & $-0,2$ & 1 & $-0,2$ & $-0,1$ & $-0,2$ & 0,2 & 0 & 0 & 0 & 0 & 0,7 & 0 & $-0,1$ & 0 & 0 & 0 \\
\hline$K_{b}$ & 0,5 & 0,6 & $-0,4$ & $-0,2$ & 1 & $-0,6$ & $-0,4$ & 0,4 & 0,1 & 0,3 & 0,4 & 0 & 0 & 0,7 & 0 & 0 & 0 & 0 \\
\hline $\mathrm{m}$ & $-0,2-$ & $-0,3$ & 0,3 & $-0,1$ & $-0,6$ & 1 & 0,3 & $-0,2$ & $-0,3$ & $-0,1$ & 0 & 0 & $-0,1$ & 0 & 0,7 & 0 & 0 & 0 \\
\hline$c(k P a)$ & $-0,2-$ & -0.2 & 0,1 & $-0,2$ & $-0,4$ & 0,3 & 1 & $-0,5$ & $-0,3$ & 0 & 0 & 0 & 0 & 0 & 0 & 0,7 & $-0,3$ & $-0,2$ \\
\hline$\varphi\left(^{(0)}\right.$ & 0,2 & 0,2 & 0,2 & 0,2 & 0,4 & $-0,2$ & -0.5 & 1 & 0,5 & 0 & 0 & 0 & 0 & 0 & 0 & $-0,3$ & 0,7 & 0.3 \\
\hline$\Delta \varphi\left(^{\circ}\right)$ & 0,4 & 0,1 & 0 & 0 & 0,1 & $-0,3$ & $-0,3$ & 0.5 & 1 & 0 & 0 & 0 & 0 & 0 & 0 & -0.2 & 0,3 & 0,7 \\
\hline K & 0,7 & 0,6 & -0.4 & 0 & 0,3 & $-0,1$ & 0 & 0 & 0 & 1 & 0,9 & $-0,7$ & $-0,1$ & 0,5 & $-0,2$ & $2-0,2$ & 0,2 & 0,4 \\
\hline $\mathrm{K}_{\mathrm{ur}}$ & 0,6 & 0,7 & $-0,4$ & 0 & 0,4 & 0 & 0 & 0 & 0 & 0,9 & 1 & $-0,6$ & $-0,1$ & 0.6 & $-0,3$ & $3-0,2$ & 0,2 & 0,1 \\
\hline $\mathrm{n}$ & $-0,4-$ & -0.4 & 0,7 & 0 & 0 & 0 & 0 & 0 & 0 & $-0,7$ & $-0,6$ & 1 & $-0,2$ & $-0,4$ & 0,3 & $\begin{array}{ll}3 & 0,1\end{array}$ & 0,2 & 0 \\
\hline $\mathrm{R}_{\text {, }}$ & 0 & 0 & 0 & 0.7 & 0 & $-0,1$ & 0 & 0 & 0 & $-0,1$ & -0.1 & $-0,2$ & 1 & $-0,2$ & $-0,1$ & $1-0,2$ & 0.2 & 0 \\
\hline$K_{\mathrm{b}}$ & 0,3 & 0,4 & 0 & 0 & 0,7 & 0 & 0 & 0 & 0 & 0.5 & 0.6 & $-0,4$ & $-0,2$ & 1 & $-0,6$ & $5-0,4$ & 0,4 & 0,1 \\
\hline $\mathrm{m}$ & $-0,1$ & 0 & 0 & $-0,1$ & 0 & 0.7 & 0 & 0 & 0 & $-0,2$ & $-0,3$ & 0,3 & $-0,1$ & -0.6 & 1 & 0,3 & $-0,2$ & $-0,3$ \\
\hline$c(k \mathrm{~Pa})$ & 0 & 0 & 0 & 0 & 0 & 0 & 0.7 & $-0,3$ & -0.2 & $-0,2$ & $-0,2$ & 0,1 & $-0,2$ & $-0,4$ & 0,3 & 31 & $-0,5$ & $-0,3$ \\
\hline$\varphi\left({ }^{2}\right)$ & 0 & 0 & 0 & 0 & 0 & 0 & $-0,3$ & 0,7 & 0,3 & 0,2 & 0,2 & 0,2 & 0,2 & 0,4 & $-0,2$ & $-0,5$ & 1 & 0,5 \\
\hline$\Delta \varphi\left({ }^{\circ}\right)$ & 0 & 0 & 0 & 0 & 0 & 0 & $-0,2$ & 0,3 & 0,7 & 0,4 & 0,1 & 0 & 0 & 0,1 & $-0,3$ & $3-0,3$ & 0,5 & 1 \\
\hline
\end{tabular}

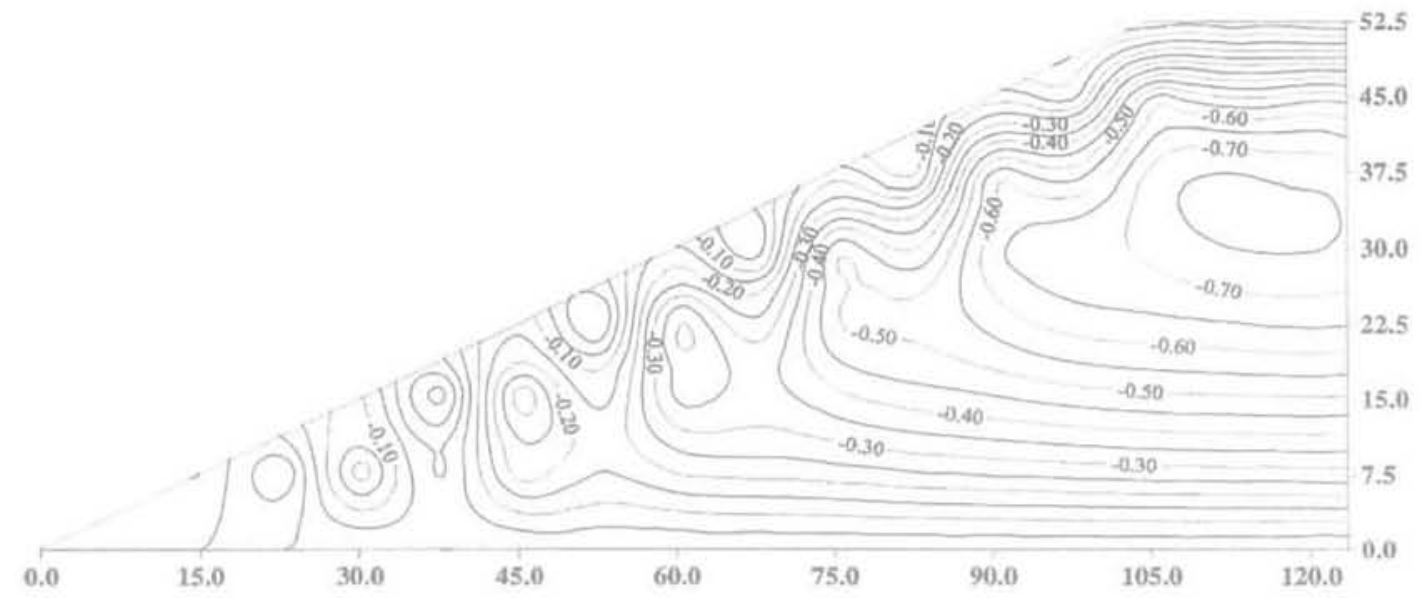

FiG.5 Déplacements verticaux (m), (espèrance mathématique),

Vertical displacements (m), (expected value)

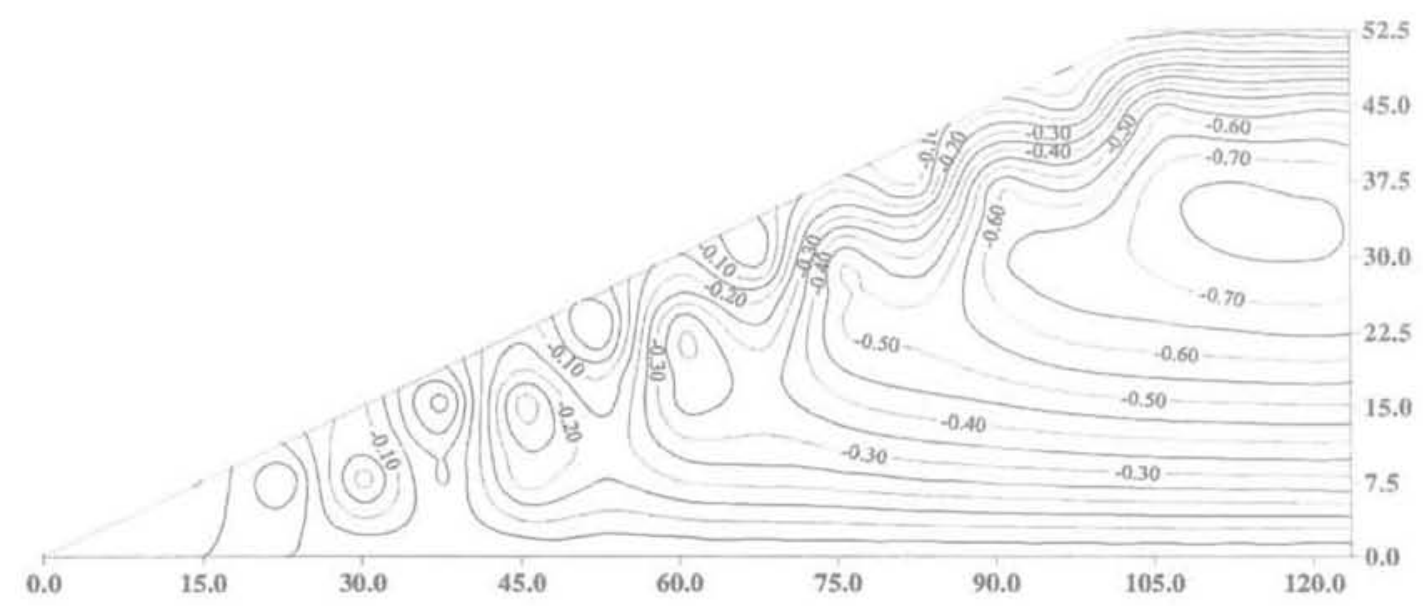

\#6.6 Coefficient de variation des déplacements verticaux (FOSM).

Coefficient of variation of vertical displacements (FOSM). 


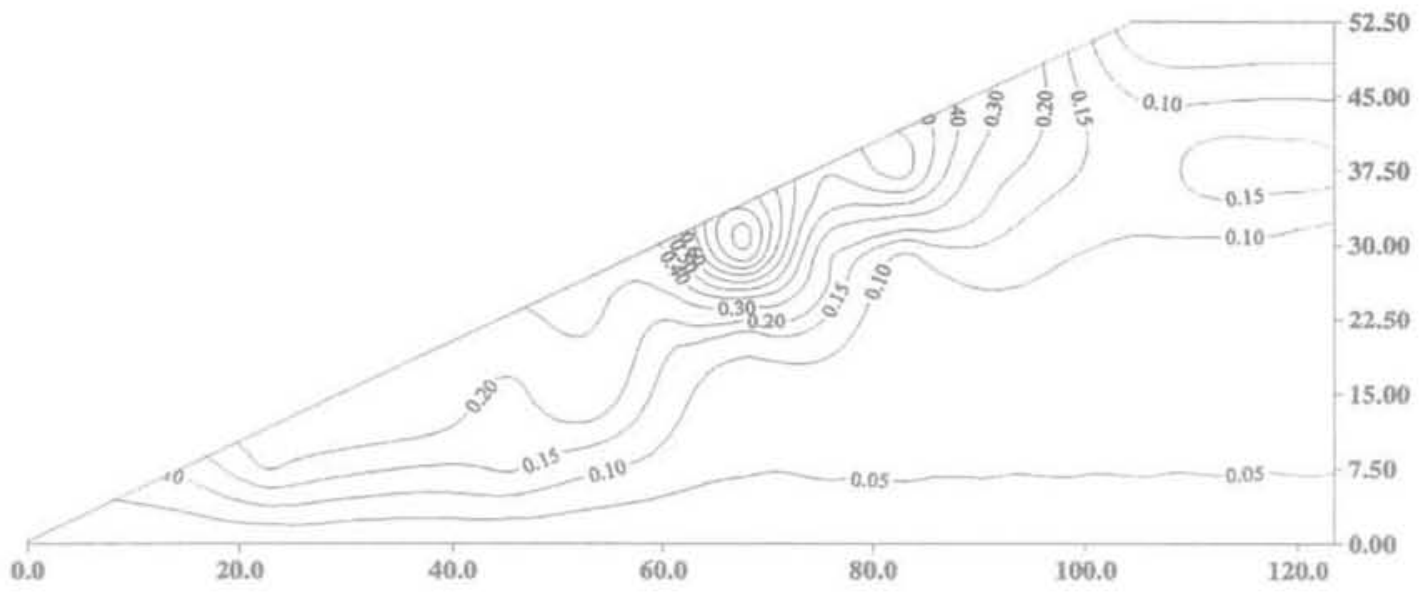

FiG. 7 Coefficient de variation des déplacements verticaux (MC),

Coefficient of variation of vertical displacements (MC).

nieurs géotechniciens d'introduire un degré de réalisme supplémentaire bien nécessaire dans les modélisations aux éléments finis. Elle est également très utile pour mettre en évidence les paramètres les plus critiques de la loi de comportement retenue pour la modélisation.

\section{Bibliographie}

Alabert F. (1997) - «The practice of fast conditional simulations through the LU decomposition of the covariance matrix w, Mathematical Geology, vol. 19, $n^{\circ} 5$, p. 369-386.

Auvinet G., Bouayed A., Ben Cheikh M. (1995) - c Modélisation stochastique par éléments finis en géornécanique $\mathrm{n}$. Actes du $12^{*}$ Congrès français de Mécanique, Strasbourg. France, vol. 4, p. 201-204.

Auvinet G. Bouayed A. Orlandi S. Lopez F. (1996) - $\alpha$ Stochastic Finite Element Method in Geomechanics s. Proceedings, Geotechnical Engineering Congress 1996, w Uncertainty 960 , University of Wisconsin. Geotechnical Engineering Division, ASCE Geotechnical Special Publication, vol. $2, n^{\circ} 58$. p. 1239-1253.

Auvinet G., Mellah R. Masrouri F. (1999) a Stochastic finite element analyses in geomechanics x. Proceedings, VIIIth International Conference on Applications of Statistics and Probability. ICASP8, Melchers \& Stewart, eds., 2000 Balkema, Rotterdarn, p. $79-85$.

Baecher G. B., Ingra T.S. (1981) - « Stochastic FEM in settlement predictions ". Journal of the Geotechnical Engineering Division. ASCE, vol. 107, $\mathrm{n}^{2}$ GT4, p. 449463

Bencheikh M. (1994) - a Eléments finis stochastiques pour les écoulements permanents x. Mémoire de DEA, ENSG. INPL, Nancy, $60 \mathrm{p}$.

Benjamin J.R., Cornell C.A. (1970) - Probability, statistics and decisions for civil engincers. New York, McGraw-Hill, p. 151-189

Benmansour A. (1996) - « Fiabilité des conduites enterrees $\mathrm{x}$. Thèse de doctorat, ENSG-INPL, Nancy, 232 p.

Bittnar Z. Sejnoha J. (1996) - $\alpha$ Numerical Methods in Structural Mechanics n. ASCE Press, Thomas Telford.

Bolle A. (1988) - " Approche probabiliste en mécanique des sols avec prise en compte de la variabilité spatiale $»$. Thèse de doctorat à l'École polytechnique fédérale de Lausanne, $n^{\circ} 743$. $265 \mathrm{p}$.

Bolle A. (1993) - " How to manage the spatial variability of natural soils $x$. Proceedings, PROBAMAT, Probabilités et matériaux, Essais, Modèles et Applications, NATO ARW930521. ENS Cachan.

Bouayed A. (1997) - $\alpha$ Modélisation stochastique par éléments finis en géomécanique w. These de doctorat, ENSG, INPL. Nancy, $157 \mathrm{p}$.

Cambou B. (1997) - « Método del elemento finito. Análisis de incertidumbre de primer orden 1 . Publicación $n^{\circ} 339$ del instituto de Ingeniería, UNAM, Mexique, $88 \mathrm{p}$.

Cambou B. (1975) - * Application of firstorder uncertainty analysis in the finite element method in linear elasticity $y$. Proceedings, Second International Conference on Application of Statistics and Probability in Soil and Structure Engineering, Aachen, p. 67-87.

Cambou B. (1975) - " L'incertitude sur les résuitats d'un problème de mécanique des sols ou des roches traitées par la méthode des éléments finis 11 . Revue française de géotechnique, $n^{\circ} 1$, p. 55-64.

Cherubini C. (1993) - a The variability of geotechnical parameters v. Probabilities and materials-Tests, Models and Applications. D. Breysse, ed. Dordrecht, Kluwer Academic Publishers, p. 69-80.

Chowdhury R.N.. Xu D.W. (1993) - « Rational polynomial technique in slope reliability analysis n. Journal of the Geotechnical Engineering Division. ASCE, vol. 119, no12, p. 1910-1928.

Cochran W.G. (1977) - Sampling techniques. John Wiley and Sons Inc.

Cornell A.C. (1971) - " First order uncertainty analysis of soils deformation and stability s. Proceedings, First International Conference on Applications of Statis- tics and Probability to Soll and Structural Engineering, Hong Kong, p. 130-144.

Deplagne F. (1994) - «Simulation de la cohésion pour une digue en argile 》. Actes $12^{\circ}$ Rencontres universitaires de génie civil, p. 75-82.

Der kiureghian A.. Ke J.B. (1988) - a The stochastic finite element method in structural reliability D. Probabilistic Engineering Mechanics, vol. A3, $n^{\circ}$ 2, p. 83-91.

Der Kiureghian A., Li C.C., Zhang $Y$, (1991) - - Recent developments in stochastic finite elements $v$. Proceedings, $4^{*}$ IFIP W.G 7.5 Conference, Munich. Springer-Verlac, , p. 19-38.

Ditlevsen O., Madsen H.O, (1996) - Structural Reliability Methods. New York. John Wiley and Sons.

Duncan J.M. Seed R.B.. Wong K.S. Ozawa Y. (1984) - « FEADAM84 : a computer program for Finite element Analysis of Dams p. Research report $n^{\circ}$ SU/GT/84-03, Department of Civil Engineering. Stanford University, USA, $68 \mathrm{p}$.

Evans D.H. (1967) - $u$ An application of numerical integration techniques to statistical tolerancing 3. Technometrix. vol. $9 . n^{\circ}$ 3. p. $441-456$.

Favre J.-L, Genevois B. (1987) - a Analyse de la sécurité des fondations superficielles vis-à-vis d'un défaut de portance : effet de la variabilité spatiale des paramètres \%. Revue française de qéotechnique $n^{\circ} 39$, p. 33-40.

Fenton G.A. Vanmarcke E.H. (1990) "Simulation of random fields via local average subdivision 1. Journal of Engineering Mechanics, vol. 116, $n$ 2 8, p. 1733 1749.

Flores Macias O. (1994) - «r Modèles fiabilistes et mécaniques : éléments finis stochastiques. Mèthodes de couplage et applications 1 . Thèse doctorale, université Blaise Pascal, Clermont-Ferrand.

Flores Macias O., Lemaire M. (1997) " Éléments finis stochastiques et fiabilité i. Revue française de génie civil, vol. 1 . $n^{\circ} 2$, p. $247-284$. 
Fogli M. (1980) - $\alpha$ Lapproche de MonteCarlo dans les problèmes de sécurité : application à l'estimation probabiliste du risque de ruine des poutres hyperstatiques en béton armé soumises à des actions aléatoires statistiques $n$. Thèse de docteur ingénieur, INSA de Lyon, $217 \mathrm{p}$.

Griffiths D.V., Fenton G.A. (1993) - *. Seepage beneath water retaining structures founded on spatially random soil $n$. Géotechnique, vol. $43, n^{\circ} 4$, p. $577-587$.

Kleiber M. Duong Hien T. (1992) - The stochastic finite element method: basic perturbation technique and computer implementation. John Wiley \& Sons Ltd. $321 \mathrm{p}$.

Kondner R.L. (1963) - a Hyperbolic stressstrain response : cohesive soils $y$. Journal of the Soil Mechanics and Fondations Division, ASCE, vol. 89, $n^{\circ}$ SM1. 115 p.

Lawrence M. (1987) - "Basic random variables in finite element analysis $D$. International Journal of Numerical Methods in Engineering, 24. , p. 18491863.

Lemaire M. (1997) - "Eléments finis stochastiques et fiabilité n. Revue francaise de génie civil, vol. $1, n^{\circ} 2$, p. 248-284.

López Acosta P. (1999) - ic Flujo de agua en suelos con permeabilidad aleatoria n. Master degree thesis, DEPFL, UNAM. Mexico. D.F.

Magnan J.-P. (1987) - « Programme de calcul par la méthode des éléments finis stochastiques (PROBEF) D. Actes, Greco Géomatériaux, Aussois, France.

Magnan J.-P., Bouheraoua A. (1995) "Stochastic analysis of soil consolidation in theory and practice n. Proceedings. VIlth International Conference on Applications of Statistics and Probability, Lemaire, Favre \& Mébarki ed., Rotterdam, Balkema, p. 77-84

Magnan J.-P., Bouheraoua A. (1996) «Analyse probabiliste de la consolidation unidimensionnelle des sols s. Revue française de géotechnique, $n^{\circ} 77$, p. 19-30.

Matheron G. - (1971) \& La théorie des variables régionalisées et ses applications 1. Fasc. 5. Ecole nationale supérieure des mines, Paris.

Mathews J., Walker R.L. (1964) - Mathematical Methods of Physics. Benjamin Publishers, $2^{\circ}$ ed., p. 286-298.
Mellah R., Auvinet G.، Masrouri F. (1998) «Estimation des incertitudes sur les résultats de la modélisation par éléments finis d'un remblai n. Actes, $2^{*}$ Conférence nationale sur la Fiabilitê des matériaux et des structures " INFiab'98*, p. 209-218.

Mellah R., Auvinet G., Masrouri F. (1998) " Stochastic Finite Element Method applied to Non-linear Analysis of Embankments n. Third International Conference on Cormputational Stochastic Mechanics. CSM'98, Computationa Stochastic Mechanics, Spanos, ed., Rotterdam, Balkema, p. 531-537.

Mellah R. (1999) - « Modélisation stochastique par éléments finis en élastoplasticité appliquée à la géomécanique n. These doctorale, ENSG-INPL,Nancy. 160 p.

Mellah R., Auvinet G. Masrouri F. (1999) "Analvse probabiliste d'un remblai en terre par la simulation de Monte-Carlo 1. Récents Progrés en génie des procédés, vol. 13, p. 173-178.

Mellah F., Auvinet G., Masrouri F. (2000) « Stochastic Finite Element Method applied to Non-linear Analysis of Embankments x. Journal of Probabilistic Engineering Mechanics, vol. $15, n^{\circ} 3$. p. $251-259$.

Mestat P., Prat M. (sous la direction de) (1999) - Ouvrages en interaction. Chapitre 12 : « Indicateurs d'erreur a priori et Chapitre 13: « Estimateurs d'erreur a posteriori $x$. Paris, Hermès Science Publications, p. 797-838.

Mrabet Z. (1999) - a Fiabilité des remblais homogènes compactés $x$. Thèse de doctorat, ENSG-INPL. Nancy, $251 \mathrm{p}$

Nechnech A. (1994) - a Étude fiabiliste des murs de soutènement w. Mémoire de DEA, ENSG-INPL, Nancy, $59 \mathrm{p}$

Orlandi S. (1996) - « Mètodo del elemento finito estocástico en geotecnia : análisis no lineal \%. Master Degree thesis. DEPFI, UNAM, Mexico, 81 p.

Poiron F. Soize C. (1989) - « Simulation numérique de champs vectoriels stochastiques gaussiens homogènes et non homogènes 1. La Recherche aérospatiale, $\mathrm{n}^{\circ} 1, \mathrm{p}, 41-61$.

Righetti G., Harrop-Williams K. (1988) "Finite element analysis of random soil media ». Journal of Geotechnical Engineering, ASCE, vol, 114, $n^{\circ} 1$ p. 59-75.

Rossa. Fry. (1988) - « Exploitation des données recueillies sur la digue Aube $x$. Électricité de France, rapport interne,

Rosenblueth E. (1975) - $\alpha$ Point estimates for probability moments is. Proceedings, Nat. Acad. Sciences, USA, vol. 72 , October, p. 3812-3814.

Soulié M., Favre M., Konrad J.-M. (1983) - « Analyse géostatistique d'un noyau de barrage tel que construit ^. Canadian Geotechnical Journal, vol. 20, p. $453-467$.

Spanos P.D., Ghanem R. (1989) - « Stochastic finite element expansion for random media 8. Journal of Engineering Mechanics, 115, , p. 499-519.

Su Y.L., Wang Y.J., Stefanko R. (1969) "Finite element analysis of underground stresses utilizing stochastically simulated material properties. Rock Mechanics: theory and Practice i. Proceedings, 11th United States Symposium on Rock Mechanics, Berkeley, Calif.. p. 253-266.

Tang W.H. (1993) - * Recent developments in geotechnical reliability x. Probabilistic Methods in Geotechnical Engineering, Li \& Lo, 1993, Rotterdam Balkema. p. $3-27$.

Teigen J.G., Frangopol D.M. (1991) - « Probabilistic FEM for non-linear concrete structures. I: theory w. Journal of Structural Engineering, ASCE, vol. 117, n* 9 , p. 2674-2689.

Vanmarcke E.H., Grigoriu M. (1983) "Stochastic finite element analysis of simple beams is. Journal of Engineering Mechanics, ASCE, vol. 109، $n^{\circ} 5$, p. $1203-$ 1214

Vanmarcke E.H. (1994) - e Stochastic finite elements and experimental measure. mentsin. Probabilistic Engineering Mechanics, vol. 9, p. 103-114.

Yongjian R., Guoqing W., Derong W., Jizhong S. (1994) - a Application of stochastic finite element method to strength and stability analysis of earth dams 1). Acta Mechanica Solida Sinica, vol. $7, n^{\circ} 1$, p. 80-86. 